\title{
ON THE CHIRAL FERMIONS IN THE TWISTOR-LIKE FORMULATION OF D=10 HETEROTIC STRING
}

\author{
Dmitrij P. Sorokin们 and Mario Tonin ${ }^{\dagger}$ \\ Dipartimento di Fisica "G. Galilei" - Università di Padova \\ Istituto Nazionale di Fisica Nucleare - Sezione di Padova \\ Padova, Italy
}

DFPD $/ 93 / \mathrm{TH} / 52$

July 1993

hep-th/

\begin{abstract}
An $n=8$ worldsheet superfield action is proposed for describing chiral fermions in the twistor-like formulation of an $N=1, D=10$ heterotic superstring.
\end{abstract}

\footnotetext{
†Supported in part by the European Community Research Program "Gauge Theories, Applied Supersymmetry and Quantum Gravity" under contract CEE-SCI-CT92-0789 and by M.P.I.

${ }^{\ddagger}$ Permanent address: Kharkov Institute of Physics and Technology, Kharkov, 310108, the Ukraine e-mail address: kfti\%kfti.kharkov.ua@relay.ussr.eu.net
} 
The problem of the origin of the fermionic $\kappa$-symmetry and the related problem of the Lorentz covariant quantization of superparticle and superstring theories (see [1] and references therein) stimulated the construction of versions of these theories which would elucidate the nature of the $\kappa$-symmetry and provide a way of covariant quantization.

In Ref. [2] it was proposed to consider the $n=N(D-2)$ parameter $\kappa$-symmetry of the $N=1,2$ superparticles and superstrings in $D=(2), 3,4,6$ and 10 dimensional space-time as a manifestation of an $n$-extended worldsheet supersymmetry.

The important feature of the doubly supersymmetric approach is that the models of this kind naturally incorporate commuting ("twistor-like") spinors as superpartners of the Grassmann coordinates of the superparticles and superstrings, thus providing a ground for unifying such fundamental trends of modern theoretical physics as supersymmetry [3] and the twistor program [4, 5]. The relationship between the doubly supersymmetric [2] and the supertwistor [6] formulation of superparticle dynamics was demonstrated in [7].

Note that a Lorentz-harmonic formulation of superparticles and superstrings [8] developed in parallel to the twistor-like doubly supersymmetric formulation is closely related to the latter on the component level.

The twistor-like approach was developed in application to superparticles [9] and superstrings [10] by several groups, and different aspects of the approach were discussed in Refs. [11]. This resulted in the construction of an $n=8$ worldline superfield version of the $N=1, D=10$ superparticle [12 and $n=(0,8)$ worldsheet superfield version of the $N=1, D=10$ heterotic superstring [13, 14, 15]. Moreover an $n=(0,2), D=10$ heterotic superstring model was proved to be a way for a consistent quantization of the $N=1, D=10$ heterotic superstring with vanishing conformal anomaly [16]. Recently the $n=8$ superconformal algebra has been observed in the light-cone gauge of the ordinary formulation of the heterotic string [17]. The twistor-like $n=(1,1), N=2, D=3$ Green-Schwarz superstring formulation has been proposed in [18].

But the inclusion of chiral fermions into the twistor-like heterotic string action has remained an unsolved problem.

Chiral fermions are necessary for the heterotic string theory to be consistent at the quantum level [1], so their incorporation into an $n=8$ worldsheet superfield action is important for accomplishing the construction of the classical twistor-like heterotic string model and may give a clue to a covariant quantization of the theory.

In the present paper we consider the problem of describing chiral fermions in the twistor-like approach.

$D=10, n=8$ twistor-like heterotic string action consists of two terms.t One of them

\footnotetext{
${ }^{1}$ The reader may find the detailed analysis of the action in Ref. [14, 11, 15].
} 
is a Wess-Zumino-type term, which serves as a string tension generation term [19, 14, 15]:

$$
S_{W Z}=\int d^{2} \xi d^{8} \eta \mathrm{P}^{\mathcal{M N}}\left(\hat{B}_{\mathcal{M N}}-\partial_{\mathcal{M}} Q_{\mathcal{N}}\right)
$$

where $\xi^{ \pm}$and $\eta^{q}(q=1, \ldots, 8),(\mathcal{M N}=(+,-, q)$ are, respectively even and odd coordinates of the super worldsheet; $\mathrm{P}^{\mathcal{M N}}=(-1)^{\mathcal{M N}+1} \mathrm{P}^{\mathcal{N} \mathcal{M}}$ is a Lagrange multiplier superfield; $Q_{\mathcal{N}}$ denotes the components of the pull-back of a target-superspace one-form $Q\left(X^{m}, \Theta^{\mu}\right)$ onto the super worldsheet $\left(\left(X^{m}(\xi, \eta), \Theta^{\mu}(\xi, \eta)(m=0,1, \ldots, 9 ; \mu=1, \ldots, 16)\right.\right.$ are vector and spinor coordinates of $N=1, D=10$ target superspace, their dependence on $\xi, \eta$ defines the embedding of the worldsheet onto the target space), and $\hat{B}_{\mathcal{M N}}$ are the components of the pull-back onto the worldsheet of a two-superform

$$
\hat{B}=B+\frac{1}{8} e^{+} \wedge e^{-} \sum_{q=1}^{8} E_{q}^{A} E_{q}^{B} E_{+}^{C} H_{C B A} .
$$

The supervielbein one-forms $E^{A}$ and the two-superform $B$ (with $H=d B$ ) determine the geometry of the target superspace together with SUGRA-SYM connections $\Omega_{A}^{B}(X, \Theta)$ and $A(X, \Theta)$. This geometry is characterized by constraints on the intrinsic components of the background torsion and curvatures [20, 5]. Capital letters $A, B, C$ from the beginning of the alphabet denote indices corresponding to the superspace tangent to the target space, and $A=(a, \alpha)$, where $a$ denotes vector and $\alpha$ denotes spinor indices. $e^{\mathcal{A}}(\mathcal{A}=+,-, q)$ are the supervielbeins on the worldsheet, the geometry of the latter being characterized by the constraints on the torsion $T^{\mathcal{A}}=\Delta e^{\mathcal{A}}(\Delta$ is a covariant differential containing Lorentz and $S O(8)$ connection) [13:

$$
T^{-}=\sum_{q=1}^{8} e^{q} \wedge e^{q} ; \quad T^{+}=0 ; \quad T^{q}=e^{+} \wedge e^{-} T_{+-}^{q} .
$$

$E_{\mathcal{A}}^{A}$ are the intrinsic components of the pull-back of $E^{A}$.

Varying (1) over $\mathrm{P}^{\mathcal{M N}}$ we get $\hat{B}_{\mathcal{M N}}=\partial_{[\mathcal{M}} Q_{\mathcal{N})}([$,$] and ($,$) denote antisymmetrization$ and symmetrization of indices, respectively) from which it follows that the pull-back of (2) onto the worldsheet $M_{w s}$ must be a closed superform, that is:

$$
\left.d \hat{B}\right|_{M_{w s}}=0
$$

To get the consistency condition (4) one must take into account constraints on the background superfields [20, 14], eqs. (3) and a twistor constraint

$$
E_{q}^{a}(X, \Theta)=0
$$

which determines the embedding of the super worldsheet into the target superspace in such a way that the odd part of the super worldsheet lies entirely within the odd part of 
the target space. A consequence of (5) is $\delta_{q r} E_{-}^{a}=E_{q} \gamma^{a} E_{r}$, which in flat target superspace is reduced to

$$
\left.\delta_{q r} \Pi_{-}^{m} \equiv \delta_{q r}\left(\partial_{-} X^{m}-i \partial \Theta \gamma^{m} \Theta\right)\right|_{\eta=0}=\lambda_{q} \gamma^{m} \lambda_{r}
$$

where $\left.\lambda_{q} \equiv \partial_{q} \Theta\right|_{\eta=0}$ are commuting twistor-like spinors; and from eq. (6) one gets a Virasoro condition $\Pi_{-}^{m} \Pi_{m-}=0$.

The closure of $\hat{B}$ (eq. (田)) on the twistor constraint surface (5), (6) is nothing but a manifestation of the light-like integrability of the $N=1, D=10$ SUGRA-SYM connections along a supersurface swept by propagating superparticles and superstrings. This underlies the twistor transform in ten dimensions [5].

From the other hand the property (4) of a superform to close (called Weil triviality) was considered in the framework of SYM theories and shown to be essential for deriving consistent chiral anomaly [21].

The other ("geometro-dynamical" [18]) term of the twistor-like heterotic string action alluded above is just needed to get eq. (5):

$$
S_{G D}=\int d^{2} \xi d^{8} \eta \mathrm{P}_{a}^{q} E_{q}^{a}
$$

where $\mathrm{P}_{a}^{q}$ is a Lagrangian multiplier.

Upon getting rid of all auxiliary fields and Lagrange multipliers one may restore the $N=1$ target-space supersymmetric part of the conventional heterotic string action. This is possible due to suitable local symmetries involving transformations of the Lagrange multipliers as discussed in [14]. Note that the kinetic and the Wess-Zumino term of the conventional action follow from the Wess-Zumino-type term (1), while the crucial role of the geometro-dynamical twistor term (17) is to match the dynamics of the string and the worldsheet geometry with the geometry of the target superspace so that the light-like integrability takes place (see [13, 14] for the details).

To include the chiral fermions into the twistor-like formulation we propose to add to eq. (11) a term

$$
S_{F}=-\int d^{2} \xi d^{8} \eta \mathrm{P}^{\mathcal{M N}}\left(i e_{\mathcal{M}}^{+} \Delta_{\mathcal{N}} \Psi^{I} \Psi^{I}\right)
$$

where the first component $\left.\psi^{I} \equiv \Psi^{I}\right|_{\eta=0}(I=1, \ldots, 32)$ of the superfield $\Psi^{I}(\xi, \eta)$ corresponds to the chiral fermion field with the conformal weight $\left(-\frac{1}{2}\right)$ in the conventional heterotic string approach, and $\Delta_{\mathcal{N}}=D_{\mathcal{N}}-E_{\mathcal{N}^{B}}^{A_{B}}$ is a covariant derivative acting on $\Psi^{I}(\xi, \eta)$ in the presence of the background gauge superfield $A(X, \Theta)$. Note that, in particular, the following (anti)commutation relations for $\Delta_{\mathcal{A}} \equiv e_{\mathcal{A}}^{\mathcal{N}} \Delta_{\mathcal{N}}$

$$
\left\{\Delta_{q}, \Delta_{r}\right\}=2 \delta_{q r} \Delta_{-} ; \quad\left[\Delta_{q}, \Delta_{-}\right]=0
$$


take place on the surface of the background, worldsheet and twistor constraints, which is again a manifestation of the light-like integrability.

Similar to eq. (4) the on-shell consistency requires the pull-back onto the super worldsheet of the form $e^{+} \wedge \Delta \Psi^{I} \Psi^{I}$ to be closed:

$$
\left.d\left(e^{+} \wedge \Delta \Psi^{I} \Psi^{I}\right)\right|_{M_{w s}}=0
$$

This is achieved by taking into account constraints (3) and including into the action a "geometro-dynamical" term for $\Psi^{I}$ :

$$
S_{F G D}=\int d^{2} \xi d^{8} \eta \mathrm{K}^{r q} \Delta_{r} \Psi^{I} \Delta_{q} \Psi^{I}
$$

where $\mathrm{K}^{r q}$ is a symmetric Lagrange multiplier superfield.

Varying (9) with respect to $\mathrm{K}^{r q}$ we get

$$
\Delta_{r} \Psi^{I} \Delta_{q} \Psi^{I}=0
$$

from which, due to the positive definiteness of the quadratic form (12) and in an assumption that the solution of (12) is to be supersymmetric, we have

$$
\Delta_{r} \Psi^{I}(\xi, \eta)=0
$$

In particular, in the flat background and in the conformal gauge (when $\left\{\Delta_{q}, \Delta_{r}\right\}=2 \delta_{q r} \partial_{-}$) eq. (13) is reduced to

$$
\partial_{-} \psi^{I}=0
$$

indicating that the leading component of $\Psi^{I}(\xi, \eta)$ is indeed the chiral fermion, while all other components vanish.

Using eqs. (3),(9) and (12) one may convince oneself that eq. (10) is valid.

The conventional heterotic fermion action is restored simultaneously with the $N=1$ supersymmetric part of the heterotic string by gauge fixing the Lagrange multipliers $\mathrm{P}^{\mathcal{M N}}$ to be

$$
\mathrm{P}^{+-}=\varepsilon^{+-} \eta^{8} T
$$

with the other components being zero, where $\mathrm{T}$ is a string tension 14 .

Thus, in particular, in the conformal gauge (and $A_{M}=0$ ) we obtain the standard heterotic fermion action

$$
S_{F}=T \int d^{2} \xi i \psi^{I} \partial_{-} \psi^{I} .
$$

The only thing which remains to check is that there is a local symmetry of the Siegel type [22] which allows one to gauge away all propagating degrees of freedom of $\mathrm{K}^{r q}$. There is indeed such a symmetry, and the relevant transformations look as follows:

$$
\delta \Psi^{I}=\epsilon_{q} \Delta_{q} \Psi^{I}, \quad \delta Q_{\mathcal{M}}=i e_{\mathcal{M}}^{+} \epsilon_{q} \Delta_{q} \Psi^{I} \Psi^{I}, \quad \delta \mathrm{P}^{\mathcal{M N}}=0
$$




$$
\begin{gathered}
\delta \mathrm{K}^{r q}=-\epsilon^{(r}\left(\Delta_{s} \mathrm{~K}_{s}^{q)}+i \mathrm{P}^{\mathcal{M} q)} e_{\mathcal{M}}^{+}\right) \\
-\frac{4}{5}\left[\Delta^{(r}\left(\left(\mathrm{K}+i \mathrm{P}^{\mathcal{M}-} e_{\mathcal{M}}^{+}\right) \epsilon^{q)}\right)+\delta^{r q} \Delta_{s}\left(\left(\mathrm{~K}+i \mathrm{P}^{\mathcal{M}-} e_{\mathcal{M}}^{+}\right) \epsilon_{s}\right)\right]+\Delta_{s} \Lambda^{s r q}
\end{gathered}
$$

where $\epsilon_{q}(\xi \eta)$ and $\Lambda^{s r q}(\xi, \eta)$ are parameters of the transformations, the latter parameter being symmetric and traceless, and $\mathrm{K} \equiv \frac{1}{8} \sum_{q} \mathrm{~K}^{q q}$.

The transformations (17) (with taking into account (9)) eliminate the traceless part of $\mathrm{K}^{q r}$ and fix $\mathrm{K}$ to be a constant. Hence, $\mathrm{K}^{q r}$ are completely auxiliary degrees of freedom.

One should notice that the action term (11) can be absorbed in the Wess-Zuminotype term by a suitable shift of $P^{\mathcal{M N}}$. In that case the structure of the local symmetries is changed but there are still enough local transformations (including that similar to eq. (17)) to gauge fix $P^{\mathcal{M N}}$ to eq. (15) and a constant. However, we prefer to keep eq. (11) since then the consideration is more transparent.

Comming back to eq. (17), the fact that we gauge fix $K$ to be a constant seems may cause a problem. Substituting $K=1$ into eq. (11) retains in the action terms like

$$
\int d^{2} \xi \varepsilon^{q_{1} \ldots q_{8}} \psi_{q_{1} \ldots q_{r}} \Delta_{-} \psi_{q_{r+1} \ldots q_{8}}
$$

where $\psi_{q_{1} \ldots q_{r}}=\left.\Delta_{q_{1}} \ldots \Delta_{q_{r}} \Psi\right|_{\eta=0}$. However, eq. (12) still holds in this gauge at $\eta=0$, and eq. (13) is again valid if the solution is required to be supersymmetric. This follows from the fact that the highest component of $K$ cannot be gauged away everywhere. We meet the same situation as in 22], and the arguments proposed by Siegel to show that the constraints are maintained even when the Lagrange multipliers are gauge fixed are applied here as well.

In conclusion we have constructed the $n=8$ worldsheet superfield action for describing heterotic fermions. The action consists of the two terms, the Wess-Zumino-type term and the geometro-dynamical term, and is naturally incorporated into the $N=1, D=10$ twistor-like heterotic string action.

When restricted to the solution of eq. (12) that obeys the worldsheet supersymmetry (eq. (13)) the model reproduces the standard heterotic string at the classical level. Non supersymmetric solutions of eq. (12) contain additional propagating fields, which may enter the action with a kinetic term having the wrong sign, thus causing the problem of unitarity. A question is whether at the quantum level the physical sector of the model can be consistently singled out. So the next step is to perform a covariant quantization of the theory, which, as one may expect, is to be a highly nontrivial problem. 


\section{Acknowledgments}

D.S. is grateful to the European Community for financial support under the contract CEE-SCI-CT92-0789, the Department of Physics of the University of Padova, and, in particular, the members of the theoretical group for kind hospitality in Padova.

D.S. is grateful to I. Bandos, S. Krivonos and D. Volkov for stimulating discussion. M.T. thanks N. Berkovits for clarifying discussion on heterotic fermions. The authors are grateful to P. Pasti for interest to this work and useful discussions. 


\section{References}

[1] M. B. Green, J. H. Schwarz and E. Witten, Superstring Theory, CUP, 1987.

[2] D. P. Sorokin, V. I. Tkach and D. V. Volkov, Mod. Phys. Lett. A4 (1989) 901.

[3] Y. A. Gol'fand and E. P. Lichtman, Zhurn. Exp. Teor. Fiz. 13, 323 (1971);

D. V. Volkov and V. P. Akulov, JETP Lett. 16, 621 (1972); Sov. Theor. Math. Phys. 1839 (1974);

J. Wess and B. Zumino, Nucl. Phys. B70, 39 (1974).

[4] R. Penrose J. Math. Phys. 8 (1967) 345;

R. Penrose and M. A. H. MacCallum, Phys. Rep. 6 (1972) 241, and refs. therein;

R. Penrose, Rep. Math. Phys. 12 (1977) 65.

[5] E. Witten, Nucl. Phys. B226 (1986) 245;

L.-L. Chau and B. Milewski, Phys. Lett. 216B (1989) 330;

L.-L. Chau and C.-S. Lim, Int. J. Mod. Phys. A4 (1989) 3819:

J. A. Shapiro and C. C. Taylor, Phys. Rep. 191 (1990) 221.

[6] A. Ferber, Nucl. Phys. B132 (1978) 55;

T. Shirafuji, Progr. Theor. Phys. 70 (1983) 18;

A. K. H. Bengtsson et.al. Phys. Rev. 36D (1987) 1786;

I. Bengtsson and M. Cederwall, Nucl. Phys. B302 (1988) 81;

Y. Eisenberg and S. Solomon, Nucl. Phys. B309 (1988) 709;

M. Plyushchay, Mod. Phys. Lett. A4 (1989) 1827.

[7] D. P. Sorokin, Fortsch. der Phys. 38 (1990) 302;

A. I. Gumenchuk and D. P. Sorokin, Sov. J. Nucl. Phys. 51 (1990) 350.

[8] E. Sokatchev, Class. Quantum Grav. 4 (1988) 237;

E. Nissimiv, S. Pacheva and S. Solomon, Nucl. Phys. B296 (1998) 492; B297 (1988) 349; Int. J. Mod. Phys. A4 (1989) 737;

R. E. Kallosh and M. A. Rahmanov, Phys. Lett. 209B (1988) 233; 214B (1988) 549;

I. A. Bandos, Sov. J. Nucl. Phys. 51 (1990) 906;

I. A. Bandos and A. A. Zheltukhin, Sov. Phys. JETP Lett. 51 (1990) 547; JETP Lett. 55 (1992) 81; Phys. Lett. 261B (1991) 245; Theor. Math. Phys. 88 (1991) 358; Fortsch. der Phys. (1993) (in press) and references therein.

[9] D. P. Sorokin, V. I. Tkach, D. V. Volkov and A. A. Zheltukhin, Phys. Lett. 216B (1989) 302; 
F. Delduc and E. Sokatchev, Class. Quantum Grav. 9 (1991) 361;

E. A. Ivanov and A. A. Kapustnikov, Phys. Lett. 267B (1991) 175;

J. P. Gauntlet Phys. Lett. 272B (1991) 25;

V. A. Soroka, D. P. Sorokin, V. I. Tkach and D. V. Volkov, Int. J. Mod. Phys. A7 (1992) 5977;

A. I. Pashnev and D. P. Sorokin Class. Quantum Grav. 10 (1993) 625.

[10] D. V. Volkov and A. A. Zheltukhin, Letters in Math. Phys. 17 (1989) 141; Nucl. Phys. B335 (1990) 723;

N. Berkovits, Phys. Lett. 232B (1989)184; 241B (1990) 497; Nucl. Phys. B350 (1991) 193; B358 (1991) 169;

M. Tonin, Phys. Lett. 266B (1991) 312;

F. Delduc, E. Ivanov and E. Sokatchev, Nucl. Phys. B384 (1992) 334;

V. Chikalov and A. Pashnev, Mod. Phys. Lett. A8 (1993) 285.

[11] P. Howe and P. Townsend, Phys. Lett. 259B (1991) 285. A. S. Galperin, P. S. Howe and K. S. Stelle, Nucl. Phys. B368 (1992) 281;

F. Delduc, A. Galperin and E. Sokatchev, Ibid. B368(1992)143;

S. Aoyama, P. Pasti and M. Tonin, Phys. Lett. 283B (1992) 213;

M. Tonin, Twistor-like formulation of heterotic strings. Talk given at the X Italian Conference on General Relativity and Gravitational Physics (Bardonecchia, September 1-5, 1992) Preprint DFPD/92/TH/59, Padova, 1992.

D. Volkov, Twistors and supersymmetry. Preprint ITP UWr 818/92, Wroclaw, 1992.

[12] A. Galperin and E. Sokatchev, Phys. Rev. D46 (1992) 714.

[13] M. Tonin, Int. J. Mod. Phys. A7 (1992) 6013;

[14] F. Delduc, A. Galperin, P. Howe and E. Sokatchev, Phys. Rev. D47 (1992) 578.

[15] I. Bandos, D. Sorokin, M. Tonin and D. Volkov, Doubly supersymmetric null string and string tension generation. Preprint DFPD/93/TH/48, Padova 1993.

[16] N. Berkovits, Nucl. Phys. B379 (1992) 96; B395 (1993) 77.

[17] L. Brink, M. Cederwall and C. P. Preitschopf, N=8 superconformal algebra and the superstring. Preprint Göteborg-ITP-93-5, 1993.

[18] A. Galperin and E. Sokatchev, Preprint JHU-TIPAC-93007, BONN-HE-93-05, 1993. 
[19] J. A. De Azcàrraga, J. M. Izquierdo and P. K. Townsend, Phys. Rev. D45 (1992) 3321 ;

P. K. Townsend, Phys. Lett. 277B (1992) 285;

E. Bergshoeff, L. A. J. London and P. K.Townsend, Class. Quantum Grav. 9 (1992) 2545.

[20] B. E. W. Nilsson, Nucl. Phys. B188 (1981) 176;

P. S. Howe, H. Nicolai and A. van Proeyen, Phys. Lett. 112B (1982) 446.

[21] L. Bonora, P. Pasti and M. Tonin, Phys. Lett. 156B (1986) 191; Nucl. Phys. B261 (1985) 241; 286 (1987) 150.

[22] W. Siegel, Nucl. Phys. B238 (1984) 307. 